Acta Crystallographica Section D

\section{Biological Crystallography}

ISSN 0907-4449

\section{Willy Wriggers $¥$}

Department of Physiology and Biophysics and Institute for Computational Biomedicine, Weill Medical College of Cornell University, 1300 York Avenue, New York, NY 10065, USA

‡ Permanent address: D. E. Shaw Research, 120 West 45th Street, New York, NY 10036, USA.

Correspondence e-mail:

wriggers@biomachina.org

\title{
Conventions and workflows for using Situs
}

Situs is a modular program package for the multi-scale modeling of atomic resolution structures and low-resolution biophysical data from electron microscopy, tomography or small-angle X-ray scattering. This article provides an overview of recent developments in the Situs package, with an emphasis on workflows and conventions that are important for practical applications. The modular design of the programs facilitates scripting in the bash shell that allows specific programs to be combined in creative ways that go beyond the original intent of the developers. Several scripting-enabled functionalities, such as flexible transformations of data type, the use of symmetry constraints or the creation of two-dimensional projection images, are described. The processing of lowresolution biophysical maps in such workflows follows not only first principles but often relies on implicit conventions. Situs conventions related to map formats, resolution, correlation functions and feature detection are reviewed and summarized. The compatibility of the Situs workflow with CCP4 conventions and programs is discussed.

\section{Introduction}

Situs is a modular command-line-based open-source package written in $\mathrm{C} / \mathrm{C}++$ and is available under the GNU GPL License. Originally designed in 1998/9 to assist in the visualization and interpretation of cryo-electron microscopy (cryoEM) density maps (Wriggers et al., 1999), its scope has been extended over the years to model multi-resolution data from a variety of biophysical sources, including tomography and small-angle X-ray scattering (SAXS; Wriggers \& Chacón, $2001 b$ ). The usefulness of hybrid multi-scale methodologies that combine atomic structures with lower resolution density maps or coarse-grained models has been well established (Mendelson \& Morris, 1997; Lindert et al., 2009) and the historical evolution of Situs with application examples was reviewed in Wriggers (2010).

The focus of this paper is on practical applications, specifically on workflows and conventions that are implicit to Situs programs. Over the years, owing to the modular design, the number of possible combinations of programs has increased to a point where it has become difficult to document possible workflows in our online tutorials. Based on specific modeling tasks, some new usage examples are provided here to inspire users to experiment on their own. Also, in an effort to bridge Situs to other software, many of the implicit conventions are described for the first time.
Received 17 June 2011

Accepted 21 November 2011 
Our online tutorials (http://situs.biomachina.org) now include Unix bash-shell scripts for the automatic generation of tutorial solutions. Much of the workflow complexity originates from the command-line-based scripting that allows programs to be combined in creative ways. Fig. 1 shows a typical example. The hypothetical problem is that one would like to bring two volumetric density maps into register. The maps can be format converted $(\$ 2)$ with the map2map tool or processed with 'volume algebra' tools (\$8) such as voledit. For technical reasons, the rigid-body matching tools collage, colores and matchp(oin)t require an atomic PDB file for docking to a target map (Fig. 1). Therefore, the second map must be intermittently transformed to the atomic (PDB) format using vol $2 \mathrm{pdb}$ so it is free from the cubic lattice (for the rotation and translation in the docking). After the matching of the pseudoPDB map, it can be interpolated back into the volumetric format through projection onto the original lattice with pdb2vol.

Many of the Situs tools rely on implicit conventions for setting parameters. It is perhaps surprising to readers from the crystallographic community that important parameters of volumetric density maps such as resolution, density levels and even map formats are not strictly defined in the hybrid modeling community. For example, the surface isolevel used for visualizing a volume map is an intuitive concept that is surprisingly difficult to solve computationally. Although one can attempt to set the isolevel based on the enclosed volume
(Harpaz et al., 1994), the resolution lowering leads to a shift in density, eroding convex features and filling up concave features of the atomic structure. To prevent convex features from protruding from the low-resolution surface, the isolevel of cryo-EM maps is empirically set to enclose $120-150 \%$ of the molecular volume depending on the overall shape of the system. This is just one example where empirical 'fudge factors' trump first principles. Over time, software developers have implemented conventions for a multitude of such quantities as they were breaking new ground. Although an effort is under way to standardize such conventions (Heymann et al., 2005), it is still often necessary to investigate the source code when sharing data between different software packages. In an effort to create more transparency, the most important Situs conventions are documented here.

The remainder of this paper is organized as follows. $\$ 2$ describes the evolution of the Situs and CCP4-based map formats. $\$ 3$ exemplifies the conversion between low-resolution structure types using small-angle X-ray-related bead models. $\$ 4$ contains a comparison of resolution conventions used for multi-scale biophysical data. $\$ 5$ describes conventions for coarse-grained models used in structure matching. $\$ 6$ presents correlation-based fitting approaches and the conventions used for computing the cross-correlation. $\S 7$ presents workflows enabled by shell scripting, such as the implementation of symmetry constraints and two-dimensional projection. The paper concludes with a discussion of extended and supplemental Situs functions in $\S 8$.

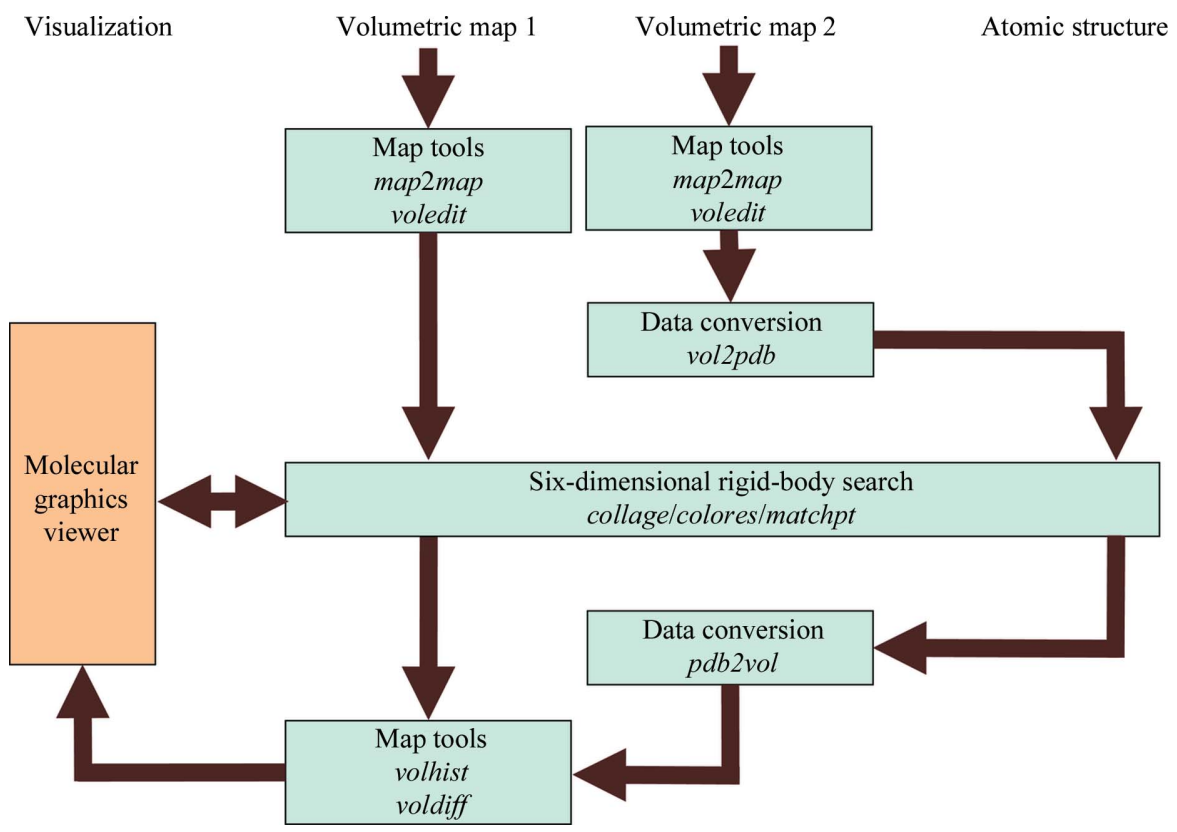

Figure 1

Modular design of the Situs package. Major Situs components (blue) are classified by their functionality. The main workflow is indicated by brown arrows. The visualization (orange) for the rendering of the models requires an external molecular-graphics viewer compatible with the density-map format, such as VMD (Humphrey et al., 1996), Chimera (Pettersen et al., 2004) or Sculptor (Birmanns et al., 2011). This example workflow shows how various Situs tools can bring two volumetric maps into register (see text). Since Situs rigid-body matching tools require a PDB file for the docking to a target map, the second map is intermittently transferred into atomic (PDB) format. After the matching of the pseudo-atomic map, it is interpolated back into volumetric format and can then be processed further.

\section{Map format conventions}

In this section, we describe threedimensional density-map formats directly supported by Situs, such as the original Situs map format and the conventions regarding various CCP4derived formats developed at MRC Cambridge, England.

Volumetric maps (Fig. 1) come in a variety of map formats, many of which were inspired by crystallographic formats. For example, the map 2 map format-conversion utility of Situs accepts cryo-EM and crystallographic density files in ASCII (text), CCP4, MRC, Situs, SPIDER and X-PLOR formats, automatically adjusts to the machine architecture (endianism) and supports permutation of axes as well as non-orthogonal unit cells (this is accomplished by trilinear interpolation to a cubic lattice, if necessary). In the late 1990s there were no universally accepted standards in the cryo-EM community for setting the origin of the map coordinate system, which is critical for the docking of atomic structures. 


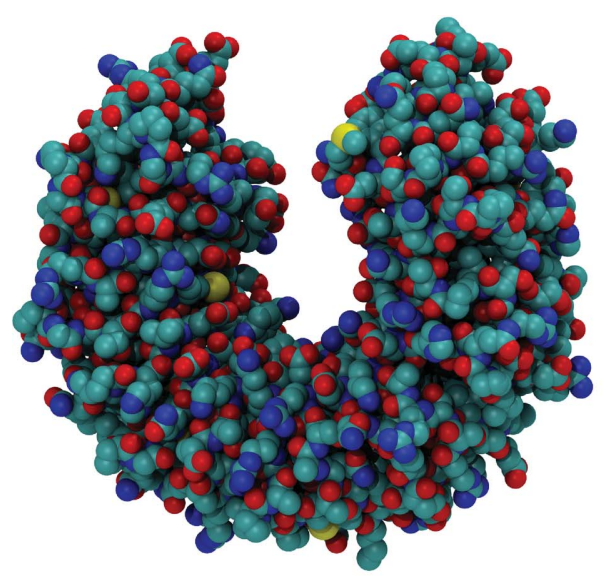

(a)

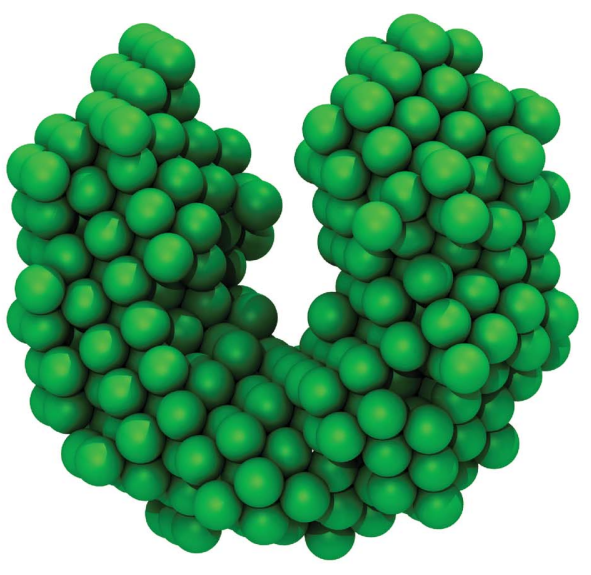

(b)

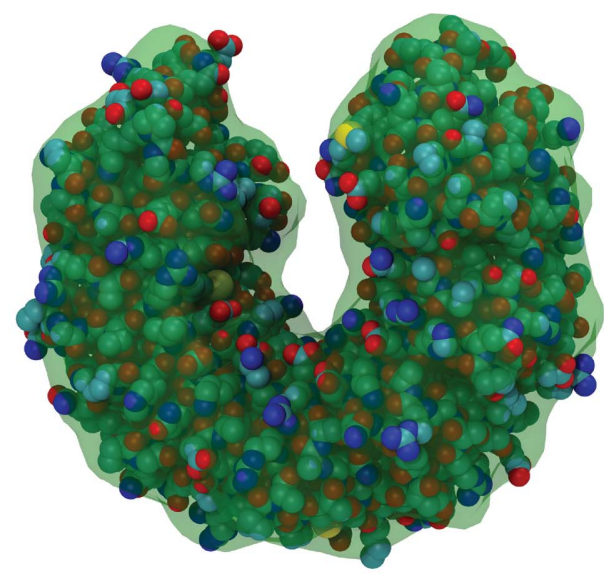

(c)

Figure 2

Real-space fitting and visualization of SAXS bead models with Situs (Wriggers \& Chacón, 2001b; Wriggers, 2010). (a) Atomic structure of ribonuclease inhibitor (PDB entry 1bnh; Kobe \& Deisenhofer, 1996). (b) Coarse-grained bead model (393 beads, 3 A radius, hexagonal close-packed) generated from (a) with the Situs tool pdb2sax. (c) Atomic structure fitted to the bead model using collage. To show the embedded structure, the bead model is rendered as a transparent envelope. The envelope is the half-maximum isosurface of a density map created from the beads with $p d b 2 v o l$ using Gaussian kernel convolution with (half-maximum) kernel radius $3 \AA$. The images were rendered with Tachyon ray tracing using VMD (Humphrey et al., 1996). For an updated review of the complete SAXS workflow, see Wriggers (2010) and the SAXS tutorial at http://situs.biomachina.org.

The minimalist Situs format was conceived to keep track of the coordinate system, to enforce a cubic lattice and to be independent of the ever-changing map-format standards in the community. Although this ASCII-based (and thus easily readable/editable) format was initially meant to be of limited use only within the Situs package, it is now supported by the molecular-graphics programs VMD (Humphrey et al., 1996), Chimera (Pettersen et al., 2004) and Sculptor (Birmanns et al., 2011), by the EMAN2 reconstruction package (Ludtke et al., 1999) and also by the em2em format-conversion tool (http:// www.imagescience.de/em2em). In the map format, a short header holds the voxel spacing WIDTH, the map origin as defined by the three-dimensional coordinates of the first voxel ORIGX, ORIGY, ORIGZ and the map dimensions (number of increments) NX, NY, NZ. This minimalist header is followed by the data fields such that $x$ increments change fastest and $z$ increments change slowest.

To take advantage of a more compact binary data storage, CCP4 and MRC file formats were recently adopted for direct use within Situs programs, eliminating the map2map formatconversion step from the workflow for many users. The rendering of the map formats was coordinated with the developers of em2em (Michael Schatz), Chimera (Tom Goddard), VMD (John Stone) and Sculptor (Stefan Birmanns). We have made sure that the Situs format matches all other format conventions, such that different map formats and PDB structures are rendered consistently. This effort required, in particular, a revisiting of the CCP4-derived map formats developed at MRC Cambridge, which have changed and diversified over the years. The MRC file format was once identical to the CCP4 format; however, incremental changes

\footnotetext{
${ }^{1}$ Supplementary material has been deposited in the IUCr electronic archive (Reference: BA5170). Services for accessing this material are described at the back of the journal.
}

and the lack of utility of some $C C P 4$ features in the EM community have caused them to become incompatible. Details of the various CCP4 and MRC map formats, and the Situs conventions for reading them, are provided as Supplementary Material $^{1}$. Our recent efforts were timely because the CCP4 format, as generated by em $2 \mathrm{em}$, has become the official format of the EMDB map data bank (Tagari et al., 2002). The detailed conventions we arrived at are described in the Supplementary Material $^{1}$ (pp. 7-8). We hope that our conventions will be more widely adopted and that they help to ensure that maps display correctly.

\section{Structure-type conversion}

Situs supports three multi-resolution structure types: atomic structures (PDB format), volumetric density maps (\$2) and SAXS bead models (PDB format). Fig. 1 shows how to interconvert between atomic and volume data.

(i) vol2pdb allows one to encode positive density values of a three-dimensional map into a PDB file, with the densities written to the PDB occupancy field.

(ii) $p d b 2 \mathrm{vol}$ is a real-space convolution tool. It allows one to lower the resolution of an atomic structure to a user-specified value or to create a bead model from atomic coordinates. The structure is first projected onto a cubic lattice using trilinear interpolation. Subsequently, each lattice point is convoluted with one of several supported kernel (point-spread) functions, e.g. Gaussian, triangular or hard sphere (see the online user guide).

Situs also supports three-dimensional bead models from SAXS (Chacón et al., 2000). Fig. 2 shows an application of SAXS-related tools in the visualization and atomic interpretation of SAXS-derived shapes (Wriggers \& Chacón, 2001b). To test the docking accuracy, the pdb2saxs tool was created, which projects atomic structures onto a hexagonal 
close-packed lattice, with user-defined bead radii written to the PDB occupancy field. The resulting models (Fig. $2 b$ ) served as 'simulated' low-resolution data in Wriggers \& Chacón (2001b).

The bead models in PDB format can be transformed into density maps for subsequent docking using a hard-sphere kernel in pdb2vol. The SAXS modeler then has access to all docking strategies supported by Situs, including correlationbased docking (collage or colores), feature-point based matching (matchpt) and even flexible real-space fitting ( $\$ 8)$.

One problem in the interpretation of SAXS data is the visualization of the beads. It is useful to render not the densely packed beads themselves (Fig. $2 b$ ), but a transparent wire mesh or envelope (Fig. 2c) that shows the fitted atomic structure. This envelope was created by isocontouring an intermediate volumetric map, which was generated from the beads by convolution with a soft kernel such as a Gaussian (using $\mathrm{pdb} 2 \mathrm{vol}$ ).

Our approach to the rendering and interpretation of SAXS data has been adopted by other groups in the structural biology community (Lipfert et al., 2007; Fagan et al., 2009; Alvarado et al., 2009). More details, including a workflow and a listing of programs that are used to dock an atomic structure into low-resolution SAXS models, are provided in an online tutorial (http://situs.biomachina.org/tutorial_saxs.html).

\section{Resolution convention}

This section relates the resolution convention used in Situs with the crystallographic resolution and the well known Rayleigh point resolution in optics.

Resolution $R$ is a quantity in Fourier space and has dimension $\AA^{-1}$, but the real-space quantity $r=R^{-1}$ is also often termed 'resolution' in biophysical parlance. The symbols $r$ and $R$ are used here to differentiate between the spatial and frequency domain.

In crystallography, let $R_{\mathrm{c}}$ be the radius of a circular region within which Fourier terms contribute to the crystallographic synthesis; one can then say that $r_{\mathrm{c}}=R_{\mathrm{c}}^{-1}$ is the crystallographic resolution (Frank, 2006). In contrast, Rayleigh considered the resolvability of two points in real space (Stenkamp \& Jensen, 1984). The real-space image of one point, whose Fourier transform is limited by a disk of radius $R_{\mathrm{c}}$, is the Airy pattern $\left[J_{1}\left(2 \pi r R_{\mathrm{c}}\right) /\left(2 \pi r R_{\mathrm{c}}\right)\right]^{2}$, where $J_{1}$ is the first-order Bessel function. The Rayleigh criterion is satisfied when the first minimum of one point's Airy pattern coincides with the central maximum of the other. This critical point-to-point distance $r_{\mathrm{p}}$ turns out to be $r_{\mathrm{p}}=0.610 r_{\mathrm{c}}$ (see Appendix $A 3$ in Radermacher, 1988). The Airy pattern is dominated by a bellshaped central Airy disk, which measures a 'full-width at halfmaximum' $\mathrm{FWHM}=0.514 r_{\mathrm{c}}$. Ignoring the weak outer rings (resulting from the hard limit in Fourier space), the Airy pattern can be approximated by a Gaussian function that matches the Airy disk (http://en.wikipedia.org/wiki/Airy_disk).

In the development of the pdb2vol tool of Situs in the 1990s, the width of the Gaussian convolution kernel was set empirically to mimic cryo-EM maps at published resolution values (for resolution estimation in cryo-EM, see Frank, 2006). The empirical Situs resolution $r_{\mathrm{s}}$ was set to $r_{\mathrm{s}}=2 \sigma$ (or 1.471 FWHM), where $\sigma$ is the three-dimensional standard deviation of the Gaussian kernel, $\exp \left(-3 r^{2} / 2 \sigma^{2}\right)$. One can relate the Situs resolution $r_{\mathrm{s}}$ to the crystallographic resolution $r_{\mathrm{c}}$ and to the point resolution $r_{\mathrm{p}}$ by matching the Gaussian to the Airy disk of the same FWHM. It follows that $r_{\mathrm{s}}=1.239 r_{\mathrm{p}}=0.756 r_{\mathrm{c}}$. Note that the Sculptor moleculargraphics software (Birmanns et al., 2011) from our laboratory also follows this convention.

The $p d b 2 m r c$ tool of the EMAN package (Ludtke et al., 1999; renamed e2pdb2mrc.py in EMAN2) is another popular resolution-lowering tool which established its own resolution measure, $r_{\mathrm{e}}$. In EMAN, the functional form of the Gaussian real-space kernel is $\exp \left(-\pi^{2} r^{2} / r_{\mathrm{e}}^{2}\right)$. The Fourier transform $\exp \left(-r_{\mathrm{e}}^{2} / R^{2}\right)$ is not strictly limited to a hard radius, as in the crystallographic case, but a reasonable radius of the soft Gaussian in Fourier space is set by EMAN at the 1/e cutoff where $R=R_{\mathrm{e}}$. After matching the Gaussian real-space kernel to the Airy disk, it follows that $r_{\mathrm{e}}=1.886 \mathrm{FWHM}=1.589 r_{\mathrm{p}}=$ $1.282 r_{\mathrm{s}}=0.969 r_{\mathrm{c}}$.

In summary, both the EMAN and Situs resolution values are larger than the point resolution and smaller than the crystallographic resolution: $r_{\mathrm{p}}<r_{\mathrm{s}}<r_{\mathrm{e}}<r_{\mathrm{c}}$. However, the Situs resolution $r_{\mathrm{s}}$ is closer to the Rayleigh criterion $r_{\mathrm{p}}$, whereas the $E M A N$ resolution $r_{\mathrm{e}}$ is almost identical to the crystallographic resolution $r_{\mathrm{c}}$ as defined by Frank (2006). When comparing similar maps between Situs (or Sculptor) and EMAN, the resolution values $r_{\mathrm{s}}$ (in units of $\AA$ ) are smaller by a factor of 1.282 compared with $r_{\mathrm{e}}$.

\section{Next-generation feature-point-based matching}

This section describes a recent paradigm shift in setting the level of detail of coarse-grained models to better represent resolved features of the structural data. Prior to Situs v.2.5, the level of detail of the coarse-grained model was limited by the algorithm, whereas now the level of detail is matched to the spatial resolution of the structures.

The idea of using coarse-grained models of feature points for the docking of multi-scale structures is a classic idea that predates Situs (Wriggers et al., 1998). We have shown in earlier work that this approach is advantageous at resolutions below $10 \AA$ because the points provide 'interior features' (and an encoding of the molecular shape) even in the absence of interior density variations from the secondary structure. However, the earlier Situs tools had a limited number of feature points and required both point clouds to be of equal size. We have recently published a new anchor-point registration technique that overcomes these combinatorial limitations and is able to dock smaller point clouds to larger ones (Birmanns \& Wriggers, 2007).

Fig. 3 shows a typical application of feature-point-based rigid-body matching. The matchpt utility is a command-line program for matching arbitrary-sized three-dimensional point sets (coarse-grained models), which can be generated on the fly or by using the output of the Situs programs $q p d b$ and qvol. 
The utility can dock a subunit into a larger target map, i.e. find $N$ feature points within another set with $M$ points, $N<M$, and match them. To solve this problem, matchpt uses a heuristic and investigates only a subset of all possible permutations of feature points (Birmanns \& Wriggers, 2007).

The idea of matching point sets was based on the observation that for many low-resolution maps numeric values of the cross-correlation (CC) are often in a narrow range and less discriminatory compared with the r.m.s.d. values of the feature points (Wriggers et al., 1999). This is a consequence of the fact that feature points can reliably and reproducibly encode the molecular shape, even in the absence of interior (secondarystructure) density features. Therefore, it makes sense for difficult low-resolution maps to use matchpt as an alternative to the CC-based tools colores and collage. In the default mode, a user would explore the quality of the match of the point clouds by minimizing their r.m.s.d. Alternatively, the minimum of the statistical variability (here the sum of average variabilities of both point sets) can be used to select an optimum $N$ and $M$, since this variability was found to be a good estimator for the docking accuracy (Wriggers \& Birmanns, 2001). Finally, a user may wish to explore the standard crosscorrelation (CC), which is discretely sampled by the solutions of the point-cloud matching.

In Situs v.2.6, the matchpt tool was improved to replace all the functionality of the classic Situs tools qdock and qrange, and the online tutorials were updated accordingly. This introduced a paradigm shift for the way the level of detail of the coarse-grained models $(N$ and $M)$ is estimated. In situations where a smaller structure is docked into a larger density (e.g. an oligomeric map), the -units parameter defines the fraction of occupied volume (which may be non-integer), i.e. it estimates how many atomic input structures fit into the target volume. $M$ is then defined as -units $\times N$. To estimate the number $N$ of feature points, one can divide the volume of the atomic structure by the volume of a resolution element, $r_{\mathrm{s}}^{3}$ (where $r_{\mathrm{s}}$ is the resolution value of the target map in $\AA$ ). This calculation gives an upper bound for the number of features $N$ contained in the structure (and, via $M$, the number of features contained in the volume) at the given map resolution. To avoid overfitting and to find an optimal number, it is useful in practical applications to bracket $N$ between the 30 to $50 \%$ level of this upper bound.

\section{Next-generation correlation-based matching}

This section highlights recent advances in correlation-based fitting technology and the underlying convention in the calculation of the CC.

The Situs cross-correlation coefficient (CC) for volumetric maps $\rho_{\text {vol }}(\mathbf{r})$ and $\rho_{\text {calc }}(\mathbf{r})$ is defined as

$$
\mathrm{CC}=\frac{\int_{V} \rho_{\mathrm{vol}}(\mathbf{r}) \times \rho_{\text {calc }}(\mathbf{r}) \mathrm{d} V}{\left[\int_{V} \rho_{\mathrm{vol}}(\mathbf{r}) \times \rho_{\mathrm{vol}}(\mathbf{r}) \mathrm{d} V\right]^{1 / 2}\left[\int_{V} \rho_{\text {calc }}(\mathbf{r}) \times \rho_{\text {calc }}(\mathbf{r}) \mathrm{d} V\right]^{1 / 2}},
$$

where $\rho_{\text {vol }}(\mathbf{r})$ is a low-resolution map and $\rho_{\text {calc }}(\mathbf{r})$ is an atomic structure subject to rigid-body movements, projection to the volume $V$ and convolution with a Gaussian kernel (Wriggers \& Chacón, 2001a). The CC values are normalized, but unlike in the Pearson correlation coefficient the averages are not subtracted from the densities. Consequently, the calculation is slightly more efficient than that of the Pearson CC. The convention takes into account that $\rho_{\text {calc }}(\mathbf{r})$ often has the physical meaning of a density with well defined positive amplitude that corresponds to the represented low-resolution structure. This way, $\mathrm{CC} \in[0,1]$ for $\rho_{\text {vol }}(\mathbf{r}), \rho_{\text {calc }}(\mathbf{r})>0$. One can show that maximizing the Situs $\mathrm{CC}$ value also maximizes the

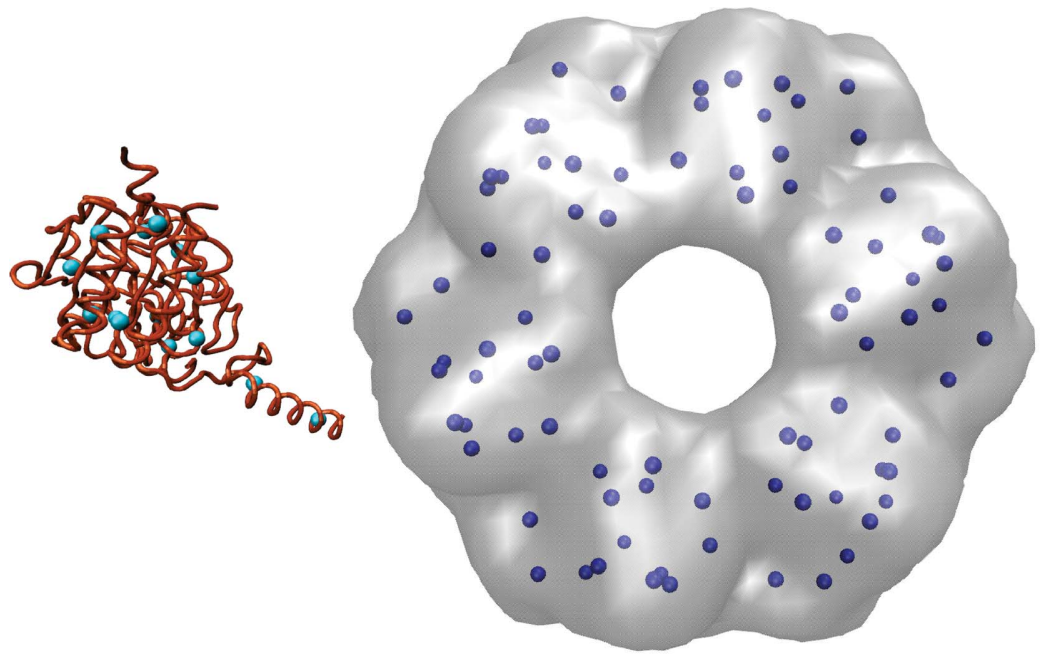

(a)

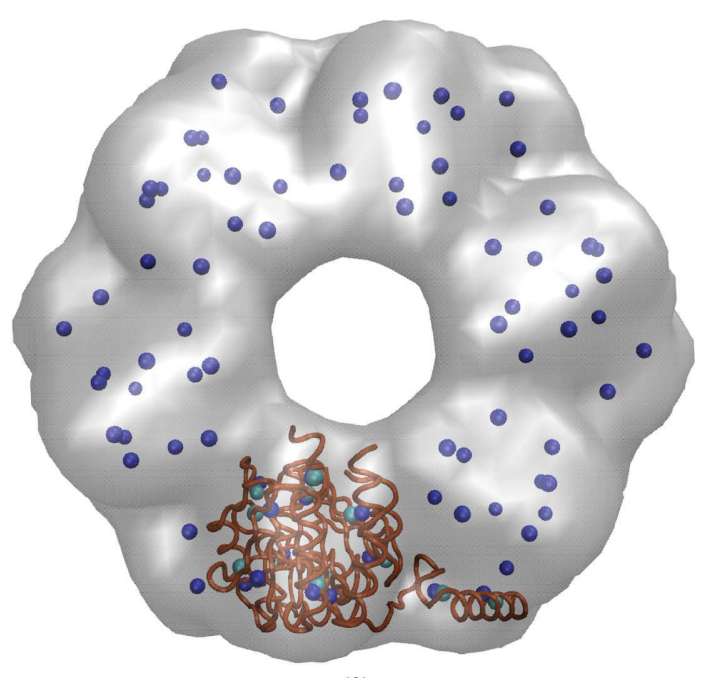

(b)

Figure 3

Rigid-body fitting of a RecA monomer (PDB entry 2rec, brown; Yu \& Egelman, 1997) to a simulated $15 \AA$ A resolution map of the hexamer (gray) using point-cloud matching. (a) Before and (b) after matching with the Situs matchpt tool. The coarse-grained anchor points (feature vectors, cyan/blue) were generated using vector quantization (Wriggers et al., 1998) in matchpt. The low-resolution map was generated with pdb2vol using a Gaussian kernel convolution. The images were rendered with $V M D$ (Humphrey et al., 1996). 
Pearson correlation coefficient, so dropping the subtraction of averages in (1) yields no performance penalty. The Situs CC convention has also been adopted by the Sculptor visualization program (Birmanns et al., 2011).

Chacón \& Wriggers (2002) introduced colores, a widely used CC-based fitting tool that takes advantage of Fourier correlation theory to rapidly scan the translational degrees of freedom of a probe molecule relative to a (fixed) targetdensity map (whereas the rotations are sampled exhaustively by the enumeration of a list of homogeneously distributed Euler angles). The performance of the standard CC (1) is limited to resolutions higher than $10 \AA$, where densities exhibit the internal (i.e. secondary) structure. The major advantage of colores is that it extends the viable resolution range to $\sim 30 \AA$ by means of an (optional) Laplacian operator [applied to both $\rho_{\text {vol }}(\mathbf{r})$ and $\rho_{\text {calc }}(\mathbf{r})$ in equation 1] that emphasizes shape-contour information in addition to the traditional volume correlation (also, a masking filter was implemented that suppresses singularities of the Laplacian of $\rho_{\mathrm{vol}}$ at density edges resulting from thresholding or segmentation).

Recently, the new refinement tool collage was introduced which performs a conjugate-gradient optimization of the same scoring functions known from colores. The main innovation is the simultaneous optimization of multiple rigid fragments that 'see' each other and avoid steric clashes by means of the normalization in (1). Birmanns et al. (2011) showed that this approach yields more accurate fits. Also, if all density is accounted for by the fragments it is no longer necessary to use the Laplacian filter option, even at low resolution.

Fig. 4 shows an application example of collage using the simultaneous optimization of six monomers. A single run of off-lattice Powell optimization is applied that refines a preliminary multi-fragment model (consisting here of six input PDB files) to the nearest maximum of the $\mathrm{CC}$. The start model of fragments could, for example, be derived manually by eye in a graphics program (as was performed here) or it could be based on colores or matchpt solutions. We have created a new multi-fragment online tutorial to explain this powerful new approach.

\section{Scripting-based workflows}

This section describes the benefits of bash-shell scripting to tie together multiple tasks. Example scripts are provided here for the implementation of symmetry constraints in multi-fragment fitting and for the creation of two-dimensional projections from three-dimensional maps.

The new collage tool and external symmetry-manipulation programs can be combined to impose symmetry constraints on the fragments during multi-fragment docking. Fig. 5 provides an overview of the workflow. Standard volumetric map formats are converted to cubic lattices in Situs format with the map2map utility. Subsequently, the volume data are inspected and, if necessary, prepared for the fitting using a variety of map tools $(\S 8)$. A data-type conversion $(\S 3)$ is optional. The fitting of multiple PDB input files to the target map is handled by collage as described in $\S 6$. Symmetry constraints are outsourced to a separate program in the Unix bash-shell script. The Situs-native pdbsymm tool allows the generation of multiple symmetry-related copies following symmetry-axis conventions based on the target map. $C, D$ and $H$ (helical) symmetry options are currently supported. For other specialized cases (e.g. crystallographic symmetry), an alternative

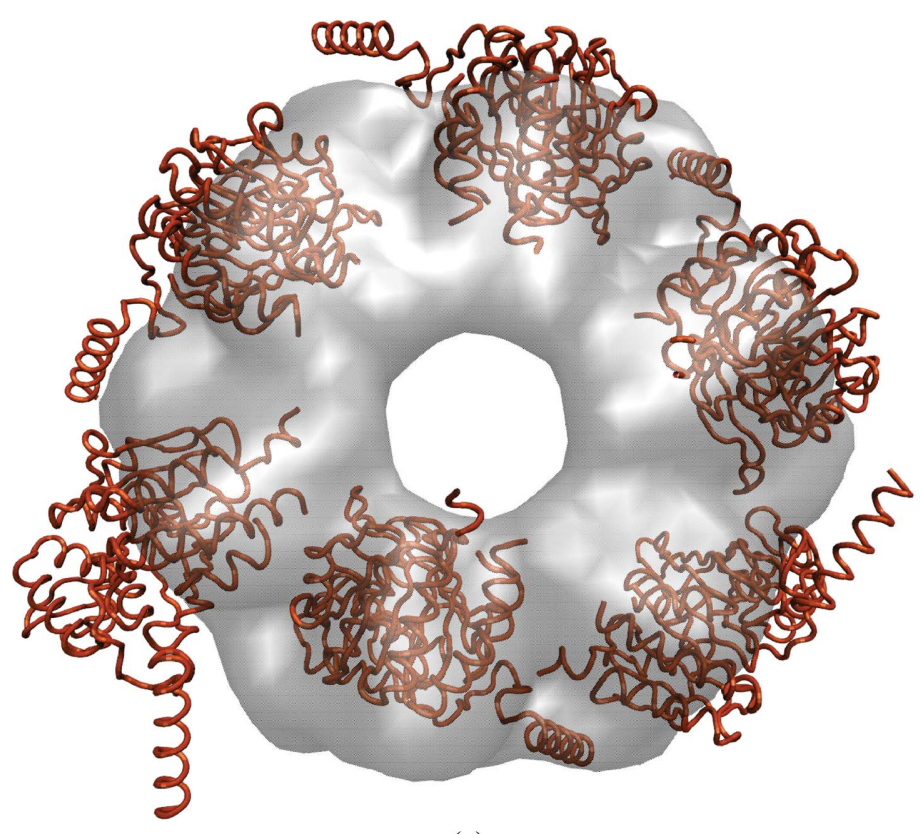

(a)

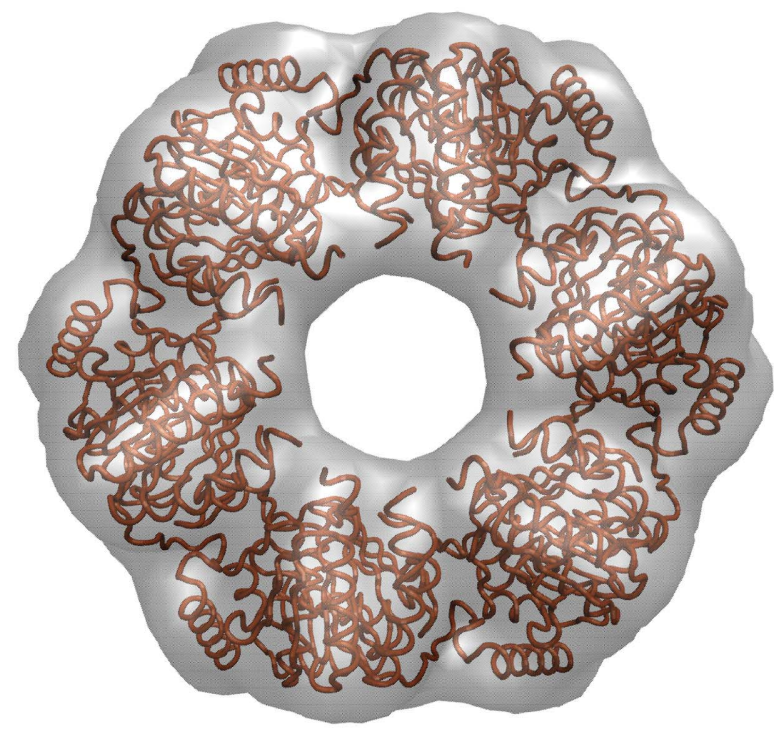

(b)

Figure 4

Multi-fragment refinement using collage. (a) RecA monomers (PDB entry 2rec, brown) in random start positions relative to the simulated $15 \AA$ resolution map of the hexamer (see Fig. 3). (b) Final fit of the monomers. The images were rendered with $V M D$ (Humphrey et al., 1996). For the complete fitting workflow, see the multi-fragment docking tutorial at http://situs.biomachina.org. 
program (such as the CCP4 pdbset tool; http://www.ccp4.ac.uk/ html/pdbset.html) may be substituted for pdbsymm in the workflow (Fig. 5). The entire Unix bash-shell implementation then proceeds as follows.

(i) Define which input structure is the initial master copy.

(ii) Generate symmetry mates from the master with pdbsymm (or alternative).

(iii) Extract individual symmetry mates from the output file using the Unix grep command (if necessary).

(iv) Refine all symmetry mates with collage using only a single conjugate-gradient step. Save master copy.

(v) Repeat (loop) steps (ii)-(iv) several times (check convergence).

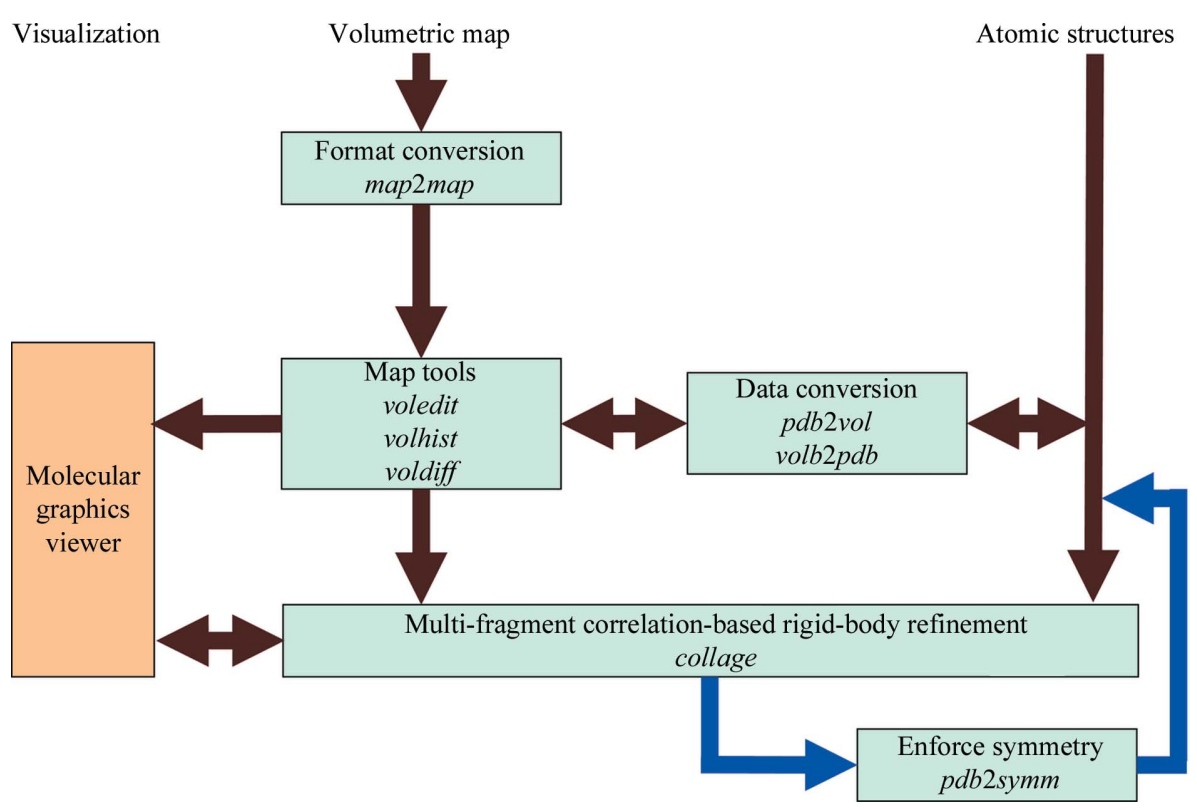

Figure 5

Schematic diagram of multiple fragment-related and symmetry-related routines in Situs (see text). The multi-fragment refinement tool collage requires one volume (target) and one or more PDB structures (probes). Symmetry constraints (blue arrows; optional) can be enforced in a bash-shell script using the pdbsymm utility or any similar tool provided by the user. The resulting docked complex can be inspected in the external graphics program. (vi) Generate final symmetry mates from the master copy using pdbsymm (or alternative).

The goal of the scripting approach is to keep Situs tools modular and to avoid having to write specialized tools for every possible symmetry scenario. This means that collage will technically still treat each fragment as independent. However, collage will take only a single conjugate-gradient step (step iv), after which the symmetry will again be enforced (in steps ii and vi). The net effect after several iterations of the loop is a symmetry-enforced conjugate-gradient optimization. An example of this approach is available online in the multifragment tutorial (file run_tutorial.bash at http:// situs.biomachina.org/tutorial_multi.html).

A new function was added to the voledit tool in Situs v.2.6 that conveniently allows the user to render twodimensional projections in addition to volume slices. Such projections are useful when comparing three-dimensional maps or resolution-lowered atomic structures with two-dimensional micrographs. Fig. 6 shows that discrepancies between two conformations can easily be detected in two dimensions. The projections (or slices) saved by voledit can be visualized by an external plotting program. Situs is mainly a three-dimensional package and does not have a tool for computing differences between two-dimensional images, but the difference of two two-dimensional projections is identical to the projection of the three-dimensional difference map, which can be computed with voldiff. The entire workflow used to create Fig. 6 consists of several steps that are most efficiently implemented in a shell script as follows.

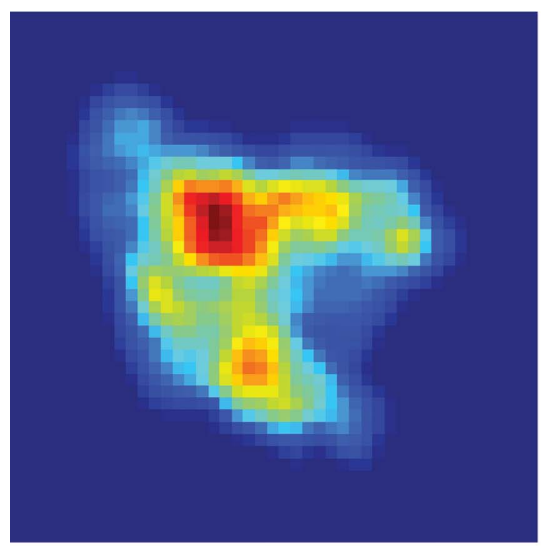

(a)

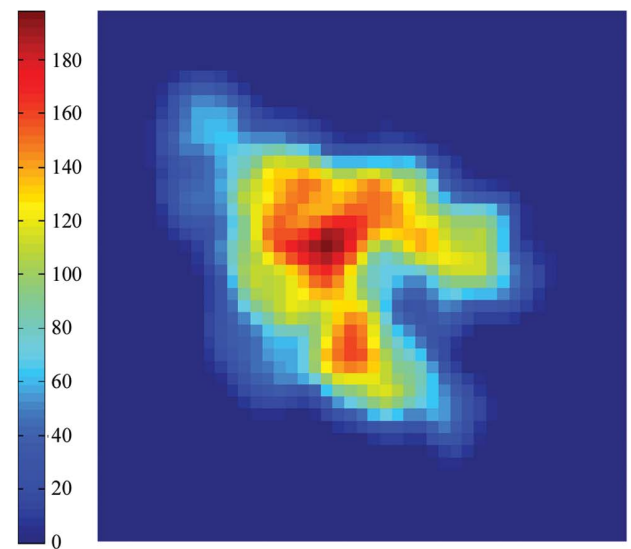

(b)

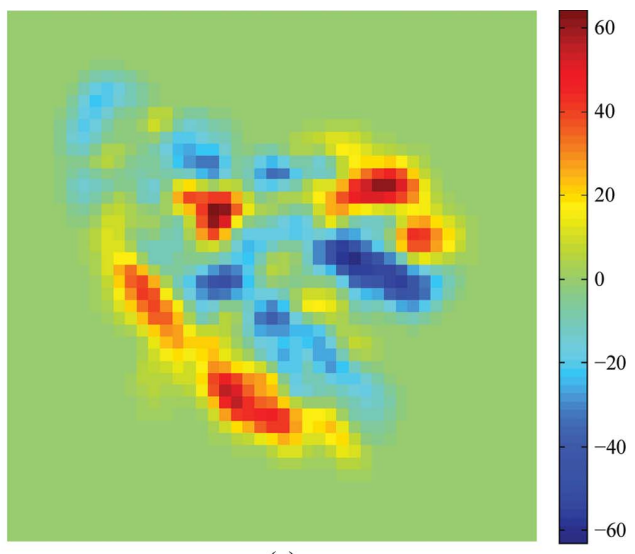

(c)

Figure 6

Two-dimensional projections and their difference. (a) Projection of a $15 \AA$ resolution map of RNA polymerase in 'open' conformation (file 0_rnap2.situs of the flexible-fitting tutorial at http://situs.biomachina.org) computed with voledit. (b) Projection of the atomic structure of RNA polymerase in 'closed' conformation (file 0_rnap1.pdb), resolution lowered to $15 \AA$ with $p d b 2 \mathrm{vol}$. (c) Projection of the difference map created with voledit and voldiff. The images were rendered with MATLAB (http://www.mathworks.com/products/matlab). 
(i) Create a low-resolution map from the atomic probe structure using $\mathrm{pdb} 2 \mathrm{vol}$.

(ii) Match the map size to that of the comparison map by cropping and zero padding using voledit.

(iii) Create the projection of both probe and comparison maps using voledit.

(iv) Create a difference map with voldiff.

(v) Create the projection of the difference map using voledit.

The shell script example (Fig. 6) is available online in part II of the flexible docking tutorial (http://situs.biomachina.org/ tutorial_flex2.html).

\section{Discussion}

The Situs software consists of multiple standalone tools that can be combined in various creative ways. The modular design offers great advantages to the user who wishes to take advantage of shell-scripting capabilities. To encourage experimentation, bash scripts are included with all online tutorials. This overview also summarizes important conventions that form the basis of Situs functionality.

One active research area that exceeds the scope of this article is flexible docking, which bring deviating features of multi-resolution structures into register (Wriggers et al., 2004; Wriggers, 2010). Fig. 6 shows the differences between flexed structures of RNA polymerase in the form of two-dimensional projections. Systematic tests and validations of flexible fitting with spatial interpolation have been carried out using the qplasty tool (Rusu et al., 2008) and experimental applications of flexible docking (using molecular-dynamics refinement) were performed in collaboration with experimental laboratories on systems such as RNA polymerase (Darst et al., 2002) and the thick filament of tarantula muscle (Alamo et al., 2008).

Another noteworthy application that exceeds the scope of this paper is 'volume algebra', in which map densities are modified according to simple algebraic operations (Wriggers et al., 2011). Volume-algebra operations are typically enabled by map-density registration (Fig. 1) and include map summation or averaging (volaver), difference mapping (voldiff), binary masking and map multiplication (voledit, volmult) and density matching using an affine transformation (volhist), as well as cropping, thresholding and segmentation (voledit). For application examples of volume algebra operations, see Fig. 1 in Wriggers et al. (2011) and the online tutorials.

The performance of multi-fragment-based refinement (Figs. 4 and 5) is the subject of ongoing research. Our empirical tests with experimental maps have shown that the refinement is very robust. The normalization of the crosscorrelation coefficient (1) penalizes steric clashes between fragments, similar to features of a tabu search using genetic algorithms (Rusu \& Birmanns, 2010). Also, the radius of convergence for the method appears to be rather large, reducing the need for exhaustive exploration. The performance will be evaluated further in future work.
Situs has been ported to multiple platforms and the source code is freely available at http://situs.biomachina.org.

A comparison of various $C C P 4$-derived map formats and related Situs read and write conventions are provided in the Supplementary Material.

I thank Michael Schatz, Mirabela Rusu, Stefan Birmanns, John Stone, Tom Goddard, Yao Cong and Steve Ludtke for helpful discussions. This work was supported in part by a grant from the National Institutes of Health (R01GM62968).

\section{References}

Alamo, L., Wriggers, W., Pinto, A., Bártoli, F., Salazar, L., Zhao, F.-Q., Craig, R. \& Padrón, R. (2008). J. Mol. Biol. 384, 780-797.

Alvarado, D., Klein, D. E. \& Lemmon, M. A. (2009). Nature (London), 461, 287-291.

Birmanns, S., Rusu, M. \& Wriggers, W. (2011). J. Struct. Biol. 173, 428-435.

Birmanns, S. \& Wriggers, W. (2007). J. Struct. Biol. 157, 271-280.

Chacón, P., Díaz, J. F., Morán, F. \& Andreu, J. M. (2000). J. Mol. Biol. 299, 1289-1302.

Chacón, P. \& Wriggers, W. (2002). J. Mol. Biol. 317, 375-384.

Darst, S., Opalka, N., Chacón, P., Polyakov, A., Richter, C., Zhang, G. \& Wriggers, W. (2002). Proc. Natl. Acad. Sci. USA, 99, 4296-4301.

Fagan, R. P., Albesa-Jové, D., Qazi, O., Svergun, D. I., Brown, K. A. \& Fairweather, N. F. (2009). Mol. Microbiol. 71, 1308-1322.

Frank, J. (2006). Three-Dimensional Electron Microscopy of Macromolecular Assemblies. Oxford University Press.

Harpaz, Y., Gerstein, M. \& Chothia, C. (1994). Structure, 2, 641-649.

Heymann, J. B., Chagoyen, M. \& Belnap, D. M. (2005). J. Struct. Biol. 151, 196-207.

Humphrey, W. F., Dalke, A. \& Schulten, K. (1996). J. Mol. Graph. 14, 33-38.

Kobe, B. \& Deisenhofer, J. (1996). J. Mol. Biol. 264, 1028-1043.

Lindert, S., Stewart, P. L. \& Meiler, J. (2009). Curr. Opin. Struct. Biol. 19, 218-225.

Lipfert, J., Das, R., Chu, V. B., Kudaravalli, M., Boyd, N., Herschlag, D. \& Doniach, S. (2007). J. Mol. Biol. 365, 1393-1406.

Ludtke, S. J., Baldwin, P. R. \& Chiu, W. (1999). J. Struct. Biol. 128, 82-97.

Mendelson, R. \& Morris, E. P. (1997). Proc. Natl. Acad. Sci. USA, 94, 8533-8538.

Pettersen, E. F., Goddard, T. D., Huang, C. C., Couch, G. S., Greenblatt, D. M., Meng, E. C. \& Ferrin, T. E. (2004). J. Comput. Chem. 25, 1605-1612.

Radermacher, M. (1988). J. Electron Microsc. Tech. 9, 359-394.

Rusu, M. \& Birmanns, S. (2010). J. Struct. Biol. 170, 164-171.

Rusu, M., Birmanns, S. \& Wriggers, W. (2008). Bioinformatics, 24, 2460-2466.

Stenkamp, R. E. \& Jensen, L. H. (1984). Acta Cryst. A40, 251-254.

Tagari, M., Newman, R., Chagoyen, M., Carazo, J. M. \& Henrick, K. (2002). Trends Biochem. Sci. 27, 589.

Wriggers, W. (2010). Biophys. Rev. 2, 21-27.

Wriggers, W., Alamo, L. \& Padrón, R. (2011). J. Struct. Biol. 173, 445450.

Wriggers, W. \& Birmanns, S. (2001). J. Struct. Biol. 133, 193-202.

Wriggers, W. \& Chacón, P. (2001a). Structure, 9, 779-788.

Wriggers, W. \& Chacón, P. (2001b). J. Appl. Cryst. 34, 773-776.

Wriggers, W., Chacón, P., Kovacs, J., Tama, F. \& Birmanns, S. (2004). Neurocomputing, 56, 365-379.

Wriggers, W., Milligan, R. A. \& McCammon, J. A. (1999). J. Struct. Biol. 125, 185-195.

Wriggers, W., Milligan, R. A., Schulten, K. \& McCammon, J. A. (1998). J. Mol. Biol. 284, 1247-1254.

Yu, X. \& Egelman, E. H. (1997). Nature Struct. Biol. 4, 101-104. 